\title{
JOINT REGISTRATION AND CHANGE DETECTION IN LONGITUDINAL BRAIN MRI
}

\author{
Eléonore Dufresne $^{1} \quad$ Denis Fortun $^{1} \quad$ Babloo Kumar $^{3} \quad$ Stéphane Kremer $^{1,2} \quad$ Vincent Noblet $^{1}$ \\ ${ }^{1}$ ICube UMR 7357, Université de Strasbourg, CNRS, Strasbourg, France \\ ${ }^{2}$ Hôpitaux Universitaires de Strasbourg, France \\ ${ }^{3}$ Indian Institute of Technology (BHU) Varanasi, India
}

\begin{abstract}
Automatic change detection in longitudinal brain MRI classically consists in a sequential pipeline where registration is estimated as a pre-processing step before detecting pathological changes such as lesion appearance. Deformable registration can advantageously be considered over rigid or affine transforms in the presence of geometrical distortions or brain atrophy to reduce false positive detections. However, this may be at the cost of underestimating the changes of interest due to the over-compensation of the differences between baseline and follow-up studies. In this article, we propose to overcome this limitation with a framework where deformable registration and lesion change are estimated jointly. We compare this joint framework with its sequential counterpart based on either affine or deformable registration. We demonstrate the benefits for detecting multiple sclerosis lesion evolutions on both synthetic and real data.
\end{abstract}

Index Terms - Longitudinal brain MRI analysis, change detection, deformable registration, optimization problem, multiple sclerosis

\section{INTRODUCTION}

Multiple Sclerosis (MS) is an auto-immune neurodegenerative disease that damages the myelin coating around the nerves through an inflammatory reaction. It is characterized by the presence of evolving lesions, together with progressive atrophy of the brain. In clinical practice, this evolution is monitored by longitudinal analysis of brain Magnetic Resonance Imaging (MRI). However, visual MRI analysis is a tedious and time-consuming task that may suffer from a lack of sensitivity and intra- and inter-observer reproducibility. Therefore, automated tools for lesion evolution assessment are necessary to alleviate these difficulties.

Most of the change detection methods rely on a sequential processing pipeline composed of two steps [1]. Firstly, the images are aligned through a registration procedure. Secondly, change detection on the registered images captures the differences due to the evolution of lesions. For the first step, many methods rely on rigid or affine registration to compensate for the difference in subject positioning only [2]. However, the deformation of the brain caused by atrophy also in- troduces changes that can hinder the identification of specific lesion progression. In particular, false detections may occur in atrophied cortical areas and around the ventricles. Using a deformable registration method can compensate accurately the atrophy, but its adverse side effect is to also compensate the evolution of lesions to minimize the dissimilarity between the images. In a sequential pipeline, this over-compensation results in underestimating the changes of interest in the detection step $[1,3]$. Note that in such a case, the characteristic behavior of the deformation field can also be used as a feature to detect the lesion changes [4], but it usually suffers from a lack of accuracy and has to be combined with intensity based techniques [5].

This paper aims to conciliate deformable registration and accurate change detection in longitudinal MRI. To this end, we propose a framework where the deformation field and the lesion changes are estimated jointly in a single minimization problem. We first recall the conventional form of the registration and change detection steps (Sec. 2). Then, we show how they can be unified in a joint optimization problem, and we detail the optimization strategy (Sec. 3). We exhibit in Sec. 4 that the proposed joint model yields better qualitative and quantitative results for detecting multiple sclerosis lesion evolution than its sequential counterpart based on either affine or deformable registration.

\section{CONVENTIONAL SEQUENTIAL APPROACH}

Let us denote $I_{1}, I_{2}: \Omega \rightarrow \mathbb{R}$ the baseline and follow-up MRI images, where $\Omega$ is the image domain. The sequential framework consists in two steps: first register $I_{2}$ onto $I_{1}$, and then detect changes between the registered images.

Registration Most registration methods can be formulated as a minimization problem of the form:

$$
\widehat{\mathbf{w}}=\underset{\mathbf{w}: \Omega \rightarrow \mathbb{R}^{3}}{\operatorname{argmin}} \sum_{\mathbf{x} \in \Omega} \rho\left(I_{1}, I_{2}, \mathbf{w}, \mathbf{x}\right)+\lambda_{1} \Psi(\mathbf{w}),
$$

where $\widehat{\mathbf{w}}$ is the deformation field between $I_{1}$ and $I_{2}$ and $\lambda_{1}>$ 0 is a tradeoff parameter. The first term is a data similarity criterion that assumes some image features to be conserved during deformation, and the second term is a regularization 
that usually promotes smooth solutions. The general formulation of (1) can be instantiated with various forms of $\rho(\cdot)$ and $\Psi(\cdot)$. An overview of deformable registration methods in medical imaging can be found in [6].

Change detection The change detection step generally consists in thresholding a map of feature differences calculated from the registered baseline and follow-up images, denoted $\rho\left(I_{1}, I_{2}, \mathbf{w}, \mathbf{x}\right)$ as in (1). Thus, the binary change map $c$ : $\Omega \rightarrow\{0,1\}$ is obtained at voxel $\mathbf{x}$ by

$$
\widehat{c}(\mathbf{x})= \begin{cases}0 & \text { if } \rho\left(I_{1}, I_{2}, \mathbf{w}, \mathbf{x}\right) \leq \lambda_{2} \\ 1 & \text { otherwise }\end{cases}
$$

where $\lambda_{2}>0$ is the detection threshold. It can be rewritten as the minimization problem:

$$
\widehat{c}(\mathbf{x})=\underset{c: \Omega \rightarrow\{0,1\}}{\operatorname{argmin}} \sum_{\mathbf{x} \in \Omega}(1-c(\mathbf{x})) \rho\left(I_{1}, I_{2}, \mathbf{w}, \mathbf{x}\right)+\lambda_{2} c(\mathbf{x}) .
$$

The most common features are either intensity-based $[1,2$, $5,7,8]$ or deformation-based [3, 5, 8] (see [9] for a survey). A simple thresholding usually leads to noisy detection maps. Therefore, most methods also include a smoothing step before or after thresholding the features map to account for neighboring spatial information.

\section{THE PROPOSED JOINT APPROACH}

\subsection{Joint model}

General formulation The limitation of the sequential framework comes from the decoupling of the two steps. Deformable registration tends to compensate the evolution of lesions while minimizing image dissimilarity, which leads to miss out on many detections. To overcome this issue, we propose to jointly estimate registration and lesion changes, by solving the unified minimization problem:

$$
\begin{array}{r}
\min _{\mathbf{w}, c} \sum_{x \in \Omega}\left[(1-c(\mathbf{x})) \rho\left(I_{1}, I_{2}, \mathbf{w}, \mathbf{x}\right)+\lambda_{2} c(\mathbf{x})\right] \\
+\lambda_{1} \Psi(\mathbf{w})+\lambda_{3} \Phi(c),
\end{array}
$$

where $\Phi(c)$ is a regularization term on $c$ and $\lambda_{3}>0$ is its associated weighting parameter. This formulation mixes the two steps of the sequential framework in a single model. The decisive advantage is that the coupling of $\mathbf{w}$ and $c$ cancels the data term in change regions, where $c(\mathbf{x})=1$, such that the estimated deformation $\mathbf{w}$ is not influenced by the presence of lesions. As a result, the deformation field is smoothed in these regions and the lesions are preserved by the registration step. Moreover, the prior on $c$ is also included in the cost function through the term $\Phi(c)$.

Specification of the cost function terms In this paper, we consider a simple special case of the general formulation (4).
We define the data term as the patch-based similarity measure:

$\rho\left(I_{1}, I_{2}, \mathbf{w}, \mathbf{x}\right)=\sum_{\mathbf{y} \in \Omega} h_{\sigma}(\mathbf{x}-\mathbf{y})\left\|I_{2}(\mathbf{y}-\mathbf{w}(\mathbf{x}))-I_{1}(\mathbf{y})\right\|_{2}^{2}$,

where $h_{\sigma}$ is a Gaussian kernel with standard deviation $\sigma$. This data term has been used for motion estimation [10] and is representative of intensity-based features of change detection methods $[2,7]$. We use a first order Tikhonov regularization of the deformation field, $\Psi(\mathbf{w})=\sum_{\mathbf{x} \in \Omega}\|\nabla \mathbf{w}(\mathbf{x})\|_{2}^{2}$, where $\nabla \cdot$ is the gradient operator, and a binary Potts model as a regularizer of $c, \Phi(c)=\sum_{\mathbf{x} \in \Omega} \sum_{\mathbf{y} \in \mathcal{N}(\mathbf{x})}(1-\delta(c(\mathbf{x})=c(\mathbf{y}))$, where $\delta$ is the Kronecker function equal to 1 if its argument is true and $\mathcal{N}(\mathbf{x})$ is the 6-neighborhood of $\mathbf{x}$.

\subsection{Optimization}

To solve (4), we rely on an alternating minimization strategy. At each iteration, we first update $\mathbf{w}$ with $c$ fixed and then update $c$ with w fixed. The joint problem is nonconvex and convergence to the global minimum cannot be guaranteed. However we detail in the sequel how the two subproblems can be efficiently solved and we show in Section 4 that we obtain satisfying results without initialization procedure.

Minimization w.r.t. w with $c$ fixed To make the problem tractable, we consider the linearized version of the data term (5) by replacing $I_{2}(\mathbf{y}-\mathbf{w}(\mathbf{x}))$ with its Taylor expansion around $\mathbf{y}$, which is a common practice in registration and motion estimation [11]. The problem becomes convex and can be addressed by a variety of efficient optimization methods. We use here the alternated direction method of multipliers (ADMM) framework. We introduce a splitting variable $\mathbf{z}$ that decouples the two terms of (4) depending on $\mathbf{w}$, and we formulate the problem in the constrained form

$$
\underset{\mathbf{w}}{\operatorname{argmin}} \sum_{x}(1-c(\mathbf{x})) \rho\left(I_{1}, I_{2}, \mathbf{w}, \mathbf{x}\right)+\lambda_{1} \Psi(\mathbf{z}), \text { s.t. } \mathbf{w}=\mathbf{z} .
$$

The ADMM algorithm is based on the minimization of the augmented Lagrangian associated with (6) w.r.t. $\mathbf{w}$ and $\mathbf{z}$, and a gradient ascent on the dual variable [12]. It leads to the following iterative updates of $\mathbf{w}$ and $\mathbf{z}$ (see [13] for a similar derivation with different data and regularization terms):

$$
\begin{aligned}
& \mathbf{w}^{k+1}=\operatorname{prox}_{\sum_{x}(1-c(\mathbf{x})) \rho\left(I_{1}, I_{2}, \cdot, \mathbf{x}\right)}\left(\mathbf{z}-\frac{\boldsymbol{\alpha}}{\mu}\right) \\
& \mathbf{z}^{k+1}=\operatorname{prox}_{\lambda_{1} \Psi}\left(\mathbf{w}+\frac{\boldsymbol{\alpha}}{\mu}\right) \\
& \boldsymbol{\alpha}^{k+1}=\boldsymbol{\alpha}^{k}+\mu\left(\mathbf{w}^{k+1}-\mathbf{z}^{k+1}\right)
\end{aligned}
$$

where $\boldsymbol{\alpha}$ is the dual variable, $\mu$ is a penalty parameter, and $\operatorname{prox}_{f}(\mathbf{x})=\underset{\mathbf{y}}{\operatorname{argmin}} \frac{1}{2}\|\mathbf{x}-\mathbf{y}\|_{2}^{2}+f(\mathbf{y})$ is the proximity operator of $f$. 
The subproblem (7) is voxel-wise and quadratic, and it admits a simple closed form solution. The subproblem (8) is equivalent to a denoising operation with the regularizer $\Psi(\cdot)$, and it also has a closed form linear solution that can be computed efficiently in the Fourier domain.

The linearization of the data term restricts the model to small deformations. Therefore, to cope with potentially large atrophy, the estimation is embedded in a coarse-to-fine scheme [14].

The main advantage of the ADMM framework for the problem at hand is to be flexible enough to cope with different registration models with low computational cost. The requirement is to be able to design a splitting of the cost function such that the proximity operators of each iteration have computationally efficient solutions. Examples of admissible models comprise data terms based on the $\ell_{1}$ penalty function or cross-correlation [15], and regularizations by total variation or Nuclear norm of the Jacobian [16].

Minimization w.r.t. $c$ with w fixed The problem (4) w.r.t. $c$ is a binary segmentation task with a submodular regularization term. It is solved exactly with a standard graph cut method [17].

\subsection{Implementation details}

We apply standard pre-processing to the images: N4 bias field correction, histogram normalization between baseline and follow-up image, and inter-study rigid registration. These steps are performed with the ANTS registration suite [18]. Since MS lesions are mostly present in the white matter, the binary change maps are masked with a white-matter union mask between baseline and follow-up time points. White matter segmentation is performed using the FSL software [19] based on either T1 or PD sequences. We initialize the alternated minimization procedure with a null deformation field and change detection map $(\mathbf{w}(\mathbf{x})=\mathbf{0}$ and $c(\mathbf{x})=0 \forall \mathbf{x} \in \Omega)$.

\section{RESULTS}

We evaluated our method on synthetic and real annotated data, and we reported the voxel-wise dice similarity coefficient (DSC) as a performance index. We compare the proposed joint approach with its sequential counterpart. We consider two types of sequential methods: sequential-def uses a deformable registration with similar ADMM optimization as in Section 3.2, and sequential-affine uses an affine registration. For all the results reported on Table 1, the hyperparameters have been chosen empirically to maximize the DSC.

\subsection{Synthetic BrainWeb data}

BrainWeb [20] is a popular database of synthetic normal and MS brains for the evaluation of brain tissue segmentation al-
Table 1. Quantitative change detection results (DSC) on synthetic and real data

\begin{tabular}{c|cc|c} 
& \multicolumn{2}{|c|}{ BrainWeb [20] } & Lesjak et al. \\
& Case 1 & Case 2 & {$[21]$} \\
\hline sequential-affine & 0.55 & 0.77 & 0.47 \\
sequential-def & 0.63 & 0.69 & 0.42 \\
joint & $\mathbf{0 . 7 0}$ & $\mathbf{0 . 7 9}$ & $\mathbf{0 . 5 2}$
\end{tabular}

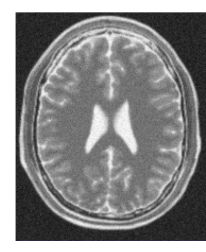

Case 1: $I_{1}$

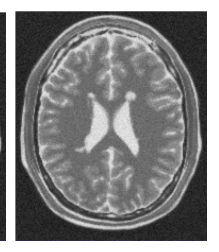

Case 1: $I_{2}$

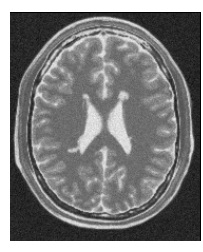

Case 2: $I_{1}$

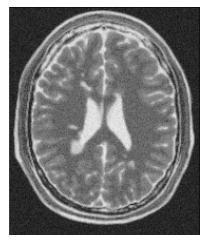

Case 2: $I_{2}$
Fig. 1. Simulated lesion evolution cases (left: Healthy brain to moderate lesion load, right: Moderate to severe lesion load).

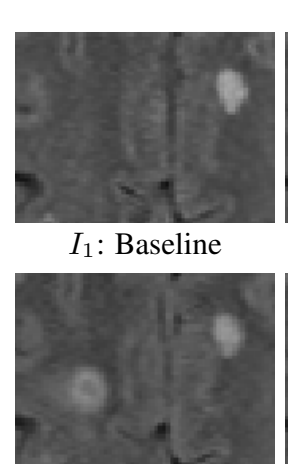

$I_{2}$ : Follow-up

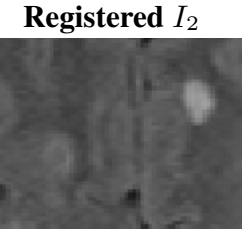

sequential-def

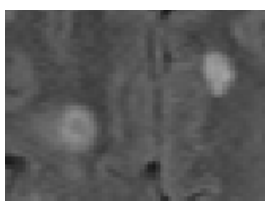

joint

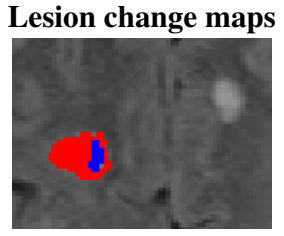

sequential-def

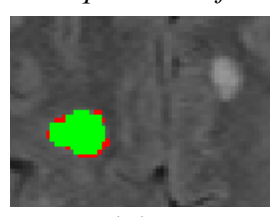

joint
Fig. 2. Comparison of the registration and lesion detection of sequential-def and joint on real data [21]. The second column shows $I_{2}$ registered on $I_{1}$ and the third column shows the lesion map superimposed on $I_{2}$. Red: Ground-truth, Blue: sequential-def, Green: joint

gorithms. We simulated images $I_{1}$ and $I_{2}$ as T2 sequences with different lesion loads. A deformation field calculated from real longitudinal MRI acquisitions was applied to $I_{2}$ to simulate atrophy. A Gaussian noise was added to all images. The variance was chosen such that the ratio between the noise and the signal of the brightest tissue equals $20 \%$. The ground-truth was obtained by thresholding at 0.3 the provided fuzzy lesion maps. Two lesion evolution cases were created (see Fig.1): Case 1 represents healthy to moderate lesion load brain models, and Case 2 represents moderate to severe lesion load.

The DSC are reported in the central columns of Table 1 for the two cases and the three compared methods. We observe that while the best performing sequential method is alternately sequential-affine or sequential-def depending on 


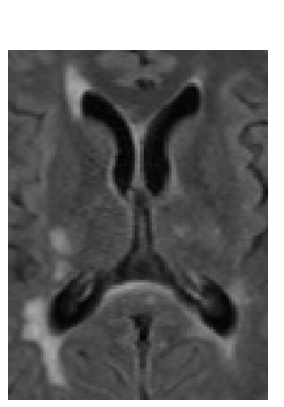

a. $I_{1}$ : Baseline

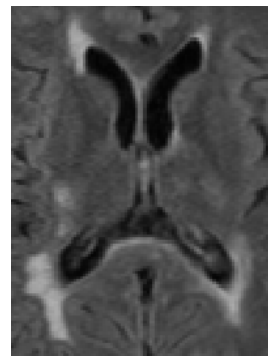

b. $I_{2}$ : Follow-up

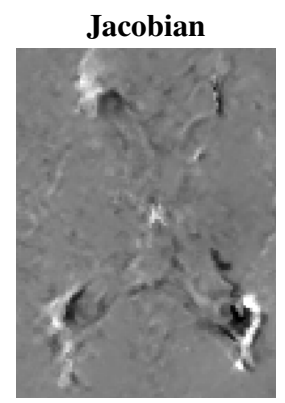

c. sequential-def

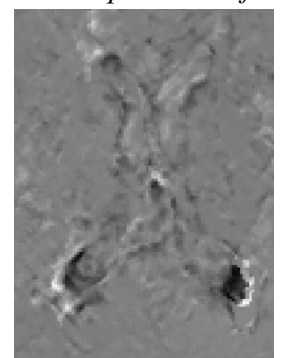

d. joint
Registered $I_{2}$

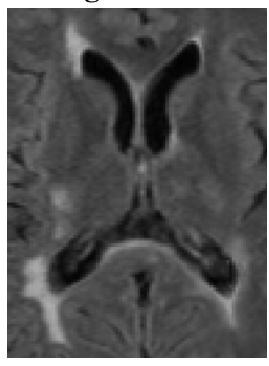

e. sequential-def

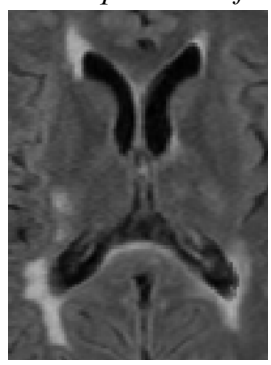

f. joint

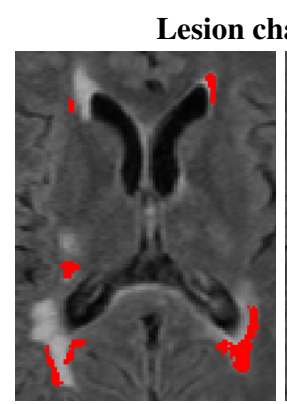

g. Ground-truth

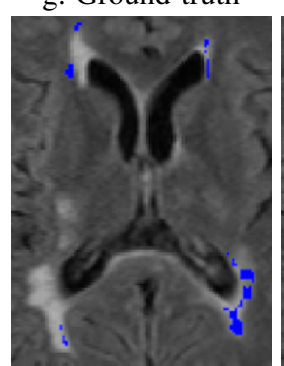

h. seqential-def

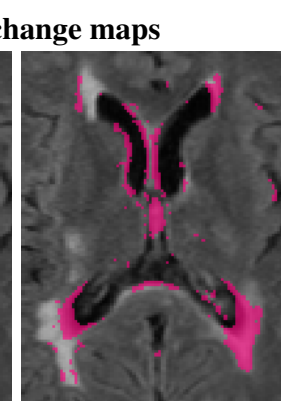

i. sequential affine

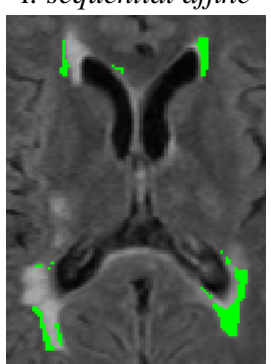

j. joint

Fig. 3. Comparison of lesion change maps obtained with the sequential affine and deformable registration pipelines and our joint deformable approach, on an example of the dataset described in [21]. The results have been obtained with the parameters maximizing the DSC.

the amount of lesions, our joint deformable approach outperforms both methods in the two cases.

\subsection{Publicly available real patients dataset}

We used the publicly available white matter change detection dataset proposed by Lesjak et al. [21] ${ }^{1}$. It consists of 20 patients, with T1w, T2w and FLAIR sequences, with anisotropic resolutions $(0.7 \times 0.7 \times 3$ and $0.9 \times 0.9 \times 3 \mathrm{~mm})$ and median time between acquisitions of 331 days. We chose to work with the FLAIR sequences, as they exhibit the most visible lesion to tissue contrast.

The median DSC values reported in the right column of Table 1 confirm the results of the synthetic case and show the superior performances of the joint method. Figure 2 illustrates how an appearing lesion can be eliminated by the registration step of sequential-def, while joint preserves the shape of the lesions and yields a more accurate change map.

In Fig.3, we analyse these differences by comparing visually the change maps of the the three methods. While sequential-affine shows good lesion coverage, false-positive regions are located around the ventricles and correspond to the detection of atrophy. The sequential-def method is not affected by atrophy, but it lacks sensitivity because of the over-compensation of lesion changes (see also Figure 2). This limitation is overcome by our joint approach that combines good sensitivity and absence of false detections due to atrophy.

\footnotetext{
${ }^{1}$ http://lit.fe.uni-lj.si/tools.php?lang=eng
}

We also show in Fig. 3 the Jacobians of the deformation fields obtained by sequential-def and joint, together with the image $I_{2}$ warped by these deformations. The darker colors in the Jacobian, which indicate diminishing tissue volume, are mainly located around the brain and ventricles due to atrophy. In the sequential approach, they are also present inside the lesions and lead to the loss of most of the lesion changes in the warped image. This explains the low sensitivity in the result of Fig.3.h. In the joint pipeline this effect is corrected and only atrophy is inverted in the warped $I_{2}$ while lesions remain unaltered.

\section{CONCLUSION}

We proposed a method that estimates jointly changes and deformation fields in longitudinal brain MRI sequences. It is particularly adapted to the monitoring of MS, where brain atrophy hinder lesion evolution detection. We demonstrated that our method combines correction of the atrophy and accurate change detection. Results on synthetic and real data illustrated the performance gain compared to the usual sequential approach. We consider here simple registration and change detection models. It has to be pointed out that the proposed framework is versatile and can be adapted to more sophisticated schemes.

Acknowledgments This work was funded by Région Grand Est and Philips Healthcare. 


\section{REFERENCES}

[1] Marcel Bosc, Fabrice Heitz, Jean-Paul Armspach, Izzie Namer, Daniel Gounot, and Lucien Rumbach, "Automatic change detection in multimodal serial MRI: application to multiple sclerosis lesion evolution," NeuroImage, vol. 20, no. 2, pp. 643656, Oct. 2003.

[2] Onur Ganiler, Arnau Oliver, Yago Diez, Jordi Freixenet, Joan C. Vilanova, Brigitte Beltran, Lluís Ramió-Torrentà, Àlex Rovira, and Xavier Lladó, "A subtraction pipeline for automatic detection of new appearing multiple sclerosis lesions in longitudinal studies," Neuroradiology, vol. 56, no. 5, pp. 363374, May 2014.

[3] David Rey, Gérard Subsol, Hervé Delingette, and Nicholas Ayache, "Automatic Detection and Segmentation of Evolving Processes in 3d Medical Images: Application to Multiple Sclerosis," p. 23.

[4] J.-P. Thirion and G. Calmon, "Deformation analysis to detect and quantify active lesions in three-dimensional medical image sequences," vol. 18, no. 5, pp. 429-441.

[5] M. Cabezas, J.F. Corral, A. Oliver, Y. Díez, M. Tintoré, C. Auger, X. Montalban, X. Lladó, D. Pareto, and À. Rovira, "Improved Automatic Detection of New T2 Lesions in Multiple Sclerosis Using Deformation Fields," American Journal of Neuroradiology, vol. 37, no. 10, pp. 1816-1823, Oct. 2016.

[6] A. Sotiras, C. Davatzikos, and N. Paragios, "Deformable Medical Image Registration: A Survey," IEEE Transactions on Medical Imaging, vol. 32, no. 7, pp. 1153-1190, July 2013.

[7] E.M. Sweeney, R.T. Shinohara, C.D. Shea, D.S. Reich, and C.M. Crainiceanu, "Automatic Lesion Incidence Estimation and Detection in Multiple Sclerosis Using Multisequence Longitudinal MRI," American Journal of Neuroradiology, vol. 34, no. 1, pp. 68-73, Jan. 2013.

[8] Mostafa Salem, Mariano Cabezas, Sergi Valverde, Deborah Pareto, Arnau Oliver, Joaquim Salvi, Àlex Rovira, and Xavier Lladó, "A supervised framework with intensity subtraction and deformation field features for the detection of new T2-w lesions in multiple sclerosis," NeuroImage: Clinical, vol. 17, pp. 607-615, 2018.

[9] Xavier Lladó, Onur Ganiler, Arnau Oliver, Robert Martí, Jordi Freixenet, Laia Valls, Joan C. Vilanova, Lluís Ramió-Torrentà, and Àlex Rovira, "Automated detection of multiple sclerosis lesions in serial brain MRI," Neuroradiology, vol. 54, no. 8, pp. 787-807, Aug. 2012.

[10] Andrés Bruhn, Joachim Weickert, and Christoph Schnörr, "Lucas/Kanade Meets Horn/Schunck: Combining Local and Global Optic Flow Methods," International Journal of Computer Vision, vol. 61, no. 3, pp. 1-21, Feb. 2005.

[11] Derek LG Hill, Philipp G Batchelor, Mark Holden, and David J Hawkes, "Medical image registration," Physics in medicine \& biology, vol. 46, no. 3, pp. R1, 2001.

[12] Stephen Boyd, Neal Parikh, Eric Chu, Borja Peleato, Jonathan Eckstein, et al., "Distributed optimization and statistical learning via the alternating direction method of multipliers," Foundations and Trends $\AA$ in Machine learning, vol. 3, no. 1, pp. 1-122, 2011.
[13] Denis Fortun, Martin Storath, Dennis Rickert, Andreas Weinmann, and Michael Unser, "Fast piecewise-affine motion estimation without segmentation," IEEE Transactions on Image Processing, vol. 27, no. 11, pp. 5612-5624, 2018.

[14] Thomas Brox, Andrés Bruhn, Nils Papenberg, and Joachim Weickert, "High accuracy optical flow estimation based on a theory for warping," in European conference on computer vision. Springer, 2004, pp. 25-36.

[15] Christoph Vogel, Stefan Roth, and Konrad Schindler, "An evaluation of data costs for optical flow," in DAGM Symposium on Pattern Recognition, 2013, pp. 343-353.

[16] Emrah Bostan, Stamatios Lefkimmiatis, Orestis Vardoulis, Nikolaos Stergiopulos, and Michael Unser, "Improved variational denoising of flow fields with application to phasecontrast mri data," IEEE Signal Processing Letters, vol. 22, no. 6, pp. 762-766, 2014.

[17] Yuri Boykov, Olga Veksler, and Ramin Zabih, "Fast approximate energy minimization via graph cuts," IEEE Transactions on pattern analysis and machine intelligence, vol. 23, no. 11, pp. 1222-1239, 2001.

[18] Brian B. Avants, Nicholas J. Tustison, Michael Stauffer, Gang Song, Baohua Wu, and James C. Gee, "The insight ToolKit image registration framework," vol. 8.

[19] Mark Jenkinson, Christian F. Beckmann, Timothy E. J. Behrens, Mark W. Woolrich, and Stephen M. Smith, "FSL," vol. 62 , no. 2 , pp. $782-790$.

[20] Chris A. Cocosco, Vasken Kollokian, Remi K.-S. Kwan, G. Bruce Pike, and Alan C. Evans, "Brainweb: Online interface to a 3d mri simulated brain database," NeuroImage, vol. 5, pp. 425, 1997.

[21] Žiga Lesjak, Franjo Pernuš, Boštjan Likar, and Žiga Špiclin, "Validation of White-Matter Lesion Change Detection Methods on a Novel Publicly Available MRI Image Database," Neuroinformatics, vol. 14, no. 4, pp. 403-420, Oct. 2016. 